\title{
Effect of Nicotine Dependence on Cognition and Quality of Life in Patients Suffering from Schizophrenia
}

\author{
Prachi Tripathi ${ }^{1}$, Avinash De Sousa ${ }^{2}$ \\ Medical College, Mumbai. \\ ${ }^{2}$ Consultant Psychiatrist and Founder Trustee, Desousa Foundation, Mumbai. \\ Corresponding author: Prachi Tripathi \\ Email - tripathiprachi05@gmail.com
}

${ }^{1}$ Clinical Psychologist, Department of Psychiatry, Sir J.J. Group of Hospitals and Government Grant

\begin{abstract}
Introduction: The purpose of the present study was to examine the impact of nicotine dependence on cognition and quality of life in patients suffering from schizophrenia. The research was carried out to see if there is any significant difference between the cognition and quality of life among patients with schizophrenia having nicotine dependence in comparison to the no nicotine consumption group.

Methodology: This study was carried out using the survey method. There were total 69 participants, all were males and the age ranged from 20 to 50 years. The participants were the outpatients visiting the government hospitals in Mumbai for their follow-ups to the psychiatrists. The data was collected from the participants after their consent was received. The screening for severity was done and only patients rated with moderate disturbances on the Positive and Negative Syndrome Scale (PANSS) were considered for further administration of tests. The two scales Montreal Cognitive Assessment Test (MoCA) and WHOQOL-Bref (Hindi) were used for measuring cognition and quality of life respectively. The obtained scores were analyzed using two simple independent samples t-test.

Results: The results showed significant difference between the scores of cognition and quality of life amongst the two groups. The study was found to be in line with the previous researches. The results indicated that cognition was significantly higher in the nicotine dependent group whereas the psychological domain of quality of life was significantly higher for the no nicotine consumption group.

Conclusion: The findings indicate the group of patients with schizophrenia having nicotine dependence was found to have significantly higher cognition but lower perceived quality of life in psychological domain, whereas the other group of patients who did not consume nicotine showed significantly higher perceived quality of life but lower cognitive functioning. Overall the obtained statistical analysis revealed significant difference between the cognition and quality of life amongst nicotine dependent group and no nicotine consumption group.
\end{abstract}

Key Words: Nicotine dependence, cognition, quality of life, schizophrenia

(Paper received $-28^{\text {th }}$ March 2018, Peer review completed $-29^{\text {th }}$ June 2018)

(Accepted $-30^{\text {th }}$ June 2018)

\section{INTRODUCTION}

Schizophrenia has been one of the most debilitating psychopathological disorders. The disorder leads to an encapsulating condition for the person suffering from schizophrenia, however presenting itself in the form of a challenging but controllable manifestation [1]. Advancements in the field of psychiatry and psychology have majorly led this population to a stage of relief not only to the patients with the ailment but also for their families. It has been always seen that a psychopathological disorder not only enervates the patients' life but it also majorly influences the life of members living with the patient. In other words, the family too suffers 
if the patient does not recover and resume to normalcy after treatment. According to World Health Organization (2016) schizophrenia is a severe mental disorder affecting more than 21 million people worldwide. WHO, 2016 has mentioned that people with schizophrenia are 2-2.5 times more likely to die early than the general population. This is often due to physical illnesses, such as cardiovascular, metabolic and infectious diseases. But it also states that schizophrenia is treatable and intervention including medicines and psychosocial support has been effective [2].

According to the National Institute of Health [3], Schizophrenia is a chronic and severe mental disorder that affects how a person thinks, feels, and behaves. People with schizophrenia may seem like they have lost touch with reality. There are three types of symptoms: Cognitive, positive and negative symptoms. NIH (2016) has given the following symptoms characterizing the disorder -

- Cognitive symptoms of schizophrenia are subtle, but for others, they are more severe and patients may notice changes in their memory or other aspects of thinking. Symptoms include poor executive functioning (the ability to understand information and use it to make decisions), trouble focusing or paying attention, problems with working memory (the ability to use information immediately after learning it)

- Positive symptoms are psychotic behaviours not generally seen in healthy people. People with positive symptoms may lose touch with some aspects of reality. Symptoms include hallucinations, delusions, thought disorders, (unusual of dysfunctional ways of thinking), movement disorders (agitated body movements).

- Negative symptoms are associated with disruptions to normal emotions and behaviors. Symptoms include, flat affect (reduced expression of emotions via facial expression or voice tone), reduced feelings of pleasure in everyday life, difficulty beginning and sustaining activities, and reduced speaking

Neisser [4] defined, cognition as 'the mental process by which external or internal input is transformed, reduced, elaborated, stored, recovered, and used. It involves a variety of functions such as perception, attention, memory, imaging, coding, planning and organization, and decision making. Such mental processes involve the generation and use of internal representations to varying degrees, and may operate independently (or not) at different stages of processing.

The disorder has its roots into the history and was earlier known as 'dementia praecox', a term given by Emil Kraepelin in the year 1893 [5]. However, earlier to Kraepelin, two German psychiatrists Kahlbaum and Hecker introduced the concept of schizophrenia in two different terms namely, 'Paraphrenia Hebetica' characterizing catatonia given by Kahlbaum in 1863 and then 'Hebephrenia' (meaning youthful sanity in Greek derived from the word 'Hebe') given by Hecker in 1871 [5]. Later Kraepelin disagreed to Hecker's description of the disorder in terms of youthful sanity that ended always in deterioration. However after several years of review Kraepelin separated dementia praecox from manic depressive insanity. Eugene Bleular argued to the concept of 'dementia praecox' as he realized that this disorder did not always end in deterioration and the patients showed improvement on receiving treatment. In the year 1911, Bleuler wrote "I call dementia praecox schizophrenia because, as I hope to show, the splitting of the different psychic functions is one of its most important features. In each case there is a more or less clear splitting of the psychological functions: as the disease becomes distinct, the personality loses its unity" [6]. The term "schizophrenia" was coined on April 24, 1908, when Professor Bleuler gave a lecture at a meeting of the German Psychiatric Association in Berlin ('schizophrenia' derived from the Greek phenomenon 'splitting of mind').

Amongst all other substances smoking has been most frequently linked to the mortality rates in people suffering from schizophrenia. It has been observed that increased smoking among people with schizophrenia has number of impacts on this population. One well-documented consequence is the increase in premature death among people with schizophrenia [7].

Though smoking may help relieve symptoms of schizophrenia, smoking also counteracts the effects of antipsychotic medication. Smoking results in faster metabolism of antipsychotics, which results in smokers being prescribed higher doses. Studies are unclear as to whether changes in smoking are caused by changes in symptoms, side effects of medication, or primary effects of medication. Different theories have proposed their views about deriving relation between smoking and the condition of suffering from schizophrenia [8]. 


\section{Biological Theories on the Relationship between Smoking and Schizophrenia}

Current theory focuses on the role of dopamine in schizophrenia, particularly how negative symptoms such as social withdrawal and apathy may be caused by a deficiency of dopamine in the prefrontal cortex while positive symptoms such as delusions and hallucinations may be caused by excess dopamine in the mesolimbic pathway. Nicotine increases release of dopamine, so it is hypothesized that smoking helps correct dopamine deficiency in the prefrontal cortex and thus relieve negative symptoms [9].

It is unclear, however, how nicotine interacts with positive symptoms, as it would follow from this theory that nicotine would exacerbate excess dopamine in the mesolimbic pathway and thus positive symptoms as well. One theory argues that the beneficial effects of nicotine on negative symptoms outweigh possible exacerbation of positive symptoms [9].

Another area of research is the role of nicotinic receptors in schizophrenia and smoking. Studies show increased numbers of exposed nicotinic receptors, which could explain the pathology of both smoking and schizophrenia. However, others argue that the increase in nicotinic receptors is a result of persistent heavy smoking, rather than schizophrenia [10].

Another source of controversy is the relationship between smoking and sensory gating in schizophrenia. Nicotine may help improve auditory gating, the ability to screen out intrusive environmental sounds. This may help improve attention spans and reduce auditory hallucinations, allowing people with schizophrenia to perceive the environment more effectively and engage in smoother motor functions. However, research shows this effect alone cannot account for increased smoking rates [11].

\section{Psychosocial Theories of Smoking in Schizophrenia}

Several psychological and social explanations have been proposed. The socioeconomic/ environmental hypothesis proposed that smoking results because many people with schizophrenia are unemployed and inactive, so smoking relieves boredom. The personality hypothesis focused on the association between smoking and higher level of neuroticism and anxiety. This hypothesis proposed that anxiety as a symptom of schizophrenia may contribute to smoking [11].

The psychological tool hypothesis argues that smokers use nicotine to manipulate their mental state in response to various environmental conditions, such as reducing stress and managing negative emotions. Research on this hypothesis notes that people with schizophrenia often cannot cope with problems in constructive ways, so use of smoking as a psychological tool may result in a vicious cycle of more and more smoking [12].

The self-medication hypothesis argues that people with schizophrenia use nicotine to deal initiate sensory gating and cognitive deficits [13]. The cognitive effects hypothesis suggests that nicotine has positive effects on cognition, so smoking is used to improve neurocognitive dysfunction.

In these hypotheses, one factor often implicated is the effects of institutionalization and boredom. However, people with schizophrenia smoke at higher rates and for longer periods than other groups that experience both institutionalization and boredom [14].

People in the general population reported that nicotine use induces relaxation and pleasurable feelings, reduces anxiety, anger, and depression. These effects may have greater importance among patients with a major mental illness because their sources of pleasure and satisfaction are likely diminished [15].

Another factor often implicated is to the side effects of antipsychotic medications. Atypical antipsychotics may work against smoking cessation, as symptoms of smoking cessation such as irritable mood, mental dulling, and increased appetite overlap with side effects of atypical antipsychotics. Some also argue that smoking works to reduce the side effects of antipsychotics. However, research shows no association between smoking and antipsychotic use after controlling for schizophrenia [16].

Yet another frequently implicated factor is increased mental acuity associated with smoking, important because of the mental dulling found over time in schizophrenia. However, both people with schizophrenia and the general population experience this effect, so it cannot fully explain increased smoking in people with schizophrenia [9]. 


\title{
METHODOLOGY
}

\author{
Hypotheses \\ Hypothesis 1 - There will be a difference in cognitive functioning between nicotine dependent group and no \\ nicotine consumption group amongst patients with schizophrenia. \\ Hypothesis 2 - There will be a difference in psychological domain of quality of life between nicotine \\ dependent group and no nicotine consumption group amongst patients with schizophrenia.
}

\section{Participants}

The sample consisted of 69 patients suffering from paranoid schizophrenia as per DSM-IV TR criteria, already diagnosed by the psychiatrist for at least one year prior to the interview. The age ranged from 20 to 50 years. Only male participants were included in the study. The data was collected at the government hospitals in Mumbai. Purposive sampling method was used to collect the data.

\section{Variables}

The research has one independent variable that is nicotine consumption in patients with schizophrenia two having two levels namely: nicotine dependent group and no nicotine consumption group among patients with schizophrenia.

The research has two dependent variables cognition and psychological domain in quality of life.

\section{Design}

The research is a survey design using questionnaires.

\section{Instruments}

1. Positive and Negative Symptoms Scale (PANSS) [17]

2. Montreal Cognitive Assessment Test (MoCA) [18]

3. WHO Quality of Life: WHOQOL-Brief Hindi Version [19]

\section{Procedure}

60 participants were purposively selected from the hospital setting when they came for their follow-up visit to their respective psychiatrists. The participant and the accompanying informant were requested to fill a consent form prior to the administration of the tests. The researcher provided information to the patient and the informant regarding the objective of the research, scales and the principle of confidentiality. The data was collected using the survey method. The first scale was a clinician rated scale for Positive and Negative Symptoms of Schizophrenia (PANSS).

Only participants obtaining mild to moderate scores on this scale were considered for further administration of Montreal Cognitive Assessment Test (MOCA) and World Health Organization's Quality of Life Scale (WHOQOL). Participants scoring more than 120 on the scale of PANSS were not included. The participants were checked for the diagnosis of nicotine dependence and were placed accordingly in one of the groups. The standardized instructions were provided before the administration. The proposed research aimed at finding that whether participants suffering from schizophrenia and having nicotine dependence showed significant difference on the performance of scales for cognition and psychological domain of quality of life in comparison to participants with no nicotine intake. The research did not aim at providing an intervention in cases of observed issues. However, identified concerns were brought to the notice and the responsible was will be informed for taking further necessary steps.

\section{RESULTS}

The result of the collected data was quantitatively analyzed. There is one independent variable that is nicotine consumption in patients with schizophrenia having two levels namely: nicotine dependent group 
and no nicotine consumption group. The dependent variables were cognition and quality of life. The scores obtained on both the tests of two different groups were taken into consideration. The inferential statistics used is independent t-test. The analysis of data was carried out using Microsoft Excel.

The descriptive statistics of the entire data revealed that the mean value of the cognition in nicotine dependent patients with schizophrenia was found to be 23.21 with a standard deviation of 5.87. The scores varied from 9 to 30 in nicotine dependent group. On the other hand, the mean value obtained for cognition in the group which did not consume nicotine was found to be significantly low that is 17.89 with a standard deviation of 3.89. Moreover, scores varied between 10 to 28 . The highest possible score on Montreal Cognitive Assessment Test is 30 whereas the minimum is 0 .

The statistical results of psychological quality of life scores show the mean of 52.40 and standard deviation of 10.39 among the nicotine dependent group with schizophrenia. The scores ranged from 31to 69 in nicotine dependent group. On the contrary the mean obtained in the group which did not consume nicotine was significantly high that is 63.86 with a standard deviation of 15.90 , where the scores ranged between 31 to 88 .

The cognitive ability for nicotine dependent group is higher in comparison to the no nicotine consumption group thus showing that nicotine has positive effect on cognitive functioning of an individual suffering from schizophrenia. The quality of life for no nicotine consumption group is higher in comparison to the nicotine dependent group in patients with schizophrenia.

Before performing the $t$ test, normality of the data was checked. Skewness and kurtosis values were examined on the total sample to check the normality of dependent variables. Results revealed the scores of cognition and psychological quality of life among patients with schizophrenia.

On the scores of cognition, the skewness and kurtosis for the nicotine dependent group was found to be 0.89 and -0.36 respectively; for no nicotine consumption group the skewness and kurtosis was found as -0.03 and -0.24 respectively.

Whereas on the score of quality of life the skewness and kurtosis for the nicotine dependent group was found to be -0.21 and -0.36 respectively; for no nicotine consumption group the skewness and kurtosis was found as -0.52 and -0.08 respectively.

The statistics obtained were -0.89 and -0.52 respectively for skewness. Similarly, for the scores of cognition and quality of life among patients with schizophrenia in the no nicotine consumption group were found to be -0.003 and -0.52 respectively for skewness. As the values remain in between the range of -1 to +1 , the data is therefore normally distributed and hence was considered for computing using t-test to find if there is a significant difference between the two groups.

The means of the two groups were compared to find if obtained difference on cognition between the groups is significant. The obtained results on $\mathrm{t}$ value are found to be $\mathrm{t}(67)=4.49, \mathrm{p}<0.001$. Thus $\mathrm{t}$-value of 4.49 is found to be significant at alpha 0.001 (two tailed) with degrees of freedom (df) as 67 .

Therefore, the hypothesis stating that there is a difference in the cognitive functioning of patients with schizophrenia having nicotine dependence than no consumption of nicotine was accepted and the null hypothesis was rejected. The means of the two groups were compared to find if obtained difference on the psychological domain in quality of life between the groups is significant. The obtained results on $t$ value are found to be $t(67)=4.49, \mathrm{p}<0.001$. The $\mathrm{t}$-value of 4.49 is found to be significant at alpha 0.001 (two tailed) with degrees of freedom (df) as 67.

Consequently, the hypothesis stating that there is a difference in psychological domain of the quality of life in patients with schizophrenia having nicotine dependence than no consumption of nicotine is retained. 


\section{DISCUSSION}

Two independent sample t-tests were computed to further elicit if the difference found between the two groups were significant or not. An independent-sample t-test was conducted on the nicotine dependent group to compare the cognition with no nicotine consumption group. Another independent-sample t-test was calculated on both the groups to compare their perceived quality of life further focusing on the psychological domain.

The first hypothesis stated that there is a difference in the cognitive functioning of patients with schizophrenia having nicotine dependence than no consumption of nicotine. The statistical result obtained indicated significant difference between the two groups as it showed that cognition was found to be better in the nicotine dependent group to that of no nicotine consumption group. Hence the data was found to be in line with the stated hypothesis for this study. The significant statistical results were also in line with the findings of the previous researches.

In a recent study on 28 smokers ( $\geq 15$ cigarettes per day), 16 outpatients who met DSM-IV criteria for schizophrenia $(n=10)$ or schizoaffective disorder $(n=6)$ and 12 community controls without a personal history of psychosis/major affective disorders were considered in the experimental sessions. Cognition was measured on visuo-spatial working memory (VSWM), sustained attention (Continuous Performance Test - Identical Pairs; CPT-IP) and prepulse inhibition (PPI) which represents sensory gating. According to the findings schizophrenia smokers (but not controls) demonstrated significantly less error on the VSWM task in the smoking relative to abstinent condition. Control (but not schizophrenia) smokers, showed evidence of CPT-IP improvement in the smoking relative to abstinent condition. However, contrary to expectation of the study, PPI in smokers with schizophrenia was unaffected by the smoking status. The results showed that smokers demonstrated significantly better performance on selected cognitive domains during typical smoking, relative to short-term (i.e. overnight) abstinence [20].

Markou and Desouza's [21] research on tobacco smoking as a preventable cause of morbidity and mortality throughout the world. The researchers found that smokers with schizophrenia were found to have higher nicotine dependent scores, they intended to experience more severe withdrawal symptoms when they were subjected to smoking cessation. Their work also indicated that patients with schizophrenia had lower cessation rates than healthy individuals. This subjected them further to significant smoking-related morbidity and unnatural or premature mortality in relation to the general population. However, the research further led to the finding that significant cholinergic functions reported in schizophrenia patients might be the reason for their compensatory action of smoking. As cholinergic neurotransmission plays an important role in cognition and is hypothesized to play an important role in schizophrenia-associated cognitive deficits. Therefore, according to this biological assumption, it can be said that smoking may lead to better the cognitive deficits that occur due to cholinergic dysfunction.

A study done by Vanable and colleagues [22] investigated whether psychiatric diagnosis, illness severity, and other substance use predicted smoking status. The study indicated that $61 \%$ of patients smoked daily, and that $18 \%$ smoked heavily (more than one pack per day). It was found that psychiatric diagnosis and illness severity contributed to increased risk for smoking, even after controlling for other substance use.

Lyon [23] found that smoking improves processing of auditory stimuli (sensory gating) by patients with schizophrenia and may lessen negative symptoms by increasing dopamine in the nucleus accumbens and the prefrontal and frontal cortex. It was also indicated that patients who smoke metabolize antipsychotics faster than non smoking patients. Hence neurobiological and psychosocial factors reinforce the high use of nicotine by patients with schizophrenia.

The previous researches therefore support the assumption found in the literature that smoking has been considered as self-medication hypothesis. The various studies have also found that smoking or nicotine consumption provides them with the relief from negative symptoms of schizophrenia.

The second hypothesis stated that there is a difference in psychological domain of the quality of life in patients with schizophrenia having nicotine dependence than no consumption of nicotine. The results obtained demonstrated that the quality of life was significantly higher in no nicotine consumption group as 
compared to the nicotine dependent group in patients diagnosed with schizophrenia. Therefore, the results found were in line with the hypothesis.

The significantly lower quality of life in the group with nicotine dependence also demonstrates that QOL is an independent factor from cognition and various other factors like ill health due to smoking dependence, loss of social support or inability to maintain relationships, or less productivity due to smoking habit, substantially low income are representative of the overall QOL.

A study conducted an assessment of social functioning and subjective quality of life in relation to symptomatic remission in schizophrenia after first psychiatric hospitalization. The long term assessment concluded that psychopathological symptoms correlated significantly with social functioning and quality of life, especially among patients without remission [24]

A cross-sectional study carried out [25] on 50 patients diagnosed with schizophrenia and with minimum duration of illness of 2 years living in community and taking regular maintenance treatment found that that quality of life was found to be negatively correlated with negative symptoms and general psychopathology. Further severity of illness was found to be negatively correlated with overall quality of life, perceived health and quality of life in environmental domain.

Connel and others [26] carried out qualitative study with a semi structured interview. The participants belonged to broad range of mental health problems at varying levels of severity. They identified seven domains important to quality of life for people with mental health problems: well-being and ill-being; relationships and a sense of belonging; activity; self- perception; autonomy, hope and hopelessness; and physical health. They found that absence of ill-being was a particularly important aspect of quality of life, especially for those with severe or chronic mental health problems. The study found a most common reply from many of the interviewees that the distress, anger and negative feelings were the most preoccupying factors in their lives. However, their findings mentioned that majority of depressive, anger and negative feelings were associated with the condition of schizophrenia as compared to other mental health illnesses.

To add further to the qualitative findings of Connel and his colleagues, another interesting research established essential deciding criteria that impacted the overall quality of life. Fujino and others [27] looked into the employment status as one of the cardinal feature that led to decreased quality of life in patients with schizophrenia. The study concluded saying that their obtained results supported the notion that better employment status enhances subjective QOL in patients with schizophrenia.

This further emphasis the fact that, stabilized patients with schizophrenia are often troubled with the negative symptoms accompanying the mental condition which requires to be addressed in terms of therapy, cognitive remediation simultaneously with medical treatment.

However, it is advised that both ends of the spectrum be incorporated so that a measure is sensitive to important changes and reflects the difficult journey to recovery of quality of life no matter the initial severity of the mental health problem. It is therefore necessary to examine empirically whether a single positive, or negative, item adequately measures the full extent of severity of any mental health condition and to develop a measure accordingly.

Lion and Diaz [28] also emphasized on their findings based on meta-analysis of 42 studies across the literature that smoking has been found to be heavily associated with schizophrenia. Not only this, but the findings also expressed pointed out that people who are about to develop schizophrenia are also more likely to take up smoking.

Thus, further research can be done on the relation between the prodromal phase and taking up smoking. Premature mortality rates in schizophrenia have been a major concern in most of the researches, reviews and studies conducted everywhere.

\section{CONCLUSION}

The purpose of the study was to examine whether there is an impact of nicotine dependence on cognition and quality of life in patients with schizophrenia in Mumbai. The inferential statistics of two independent sample t-tests were used to analyze the data. The findings indicate the group of patients with schizophrenia having nicotine dependence was found to have significantly higher cognition but lower perceived quality of life in psychological domain, whereas the other group of patients who did not consume nicotine showed 
significantly higher perceived quality of life but lower cognitive functioning. Overall the obtained statistical analysis revealed significant difference between the cognition and quality of life amongst nicotine dependent group and no nicotine consumption group.

\section{REFERENCES}

1. Insel TR. Rethinking schizophrenia. Nature 2010;468(7321):187-93.

2. World Health Organisation. Schizophrenia and Public Health. WHO: Geneva; 2014.

3. National Institute of Health. Schizophrenia. NIH: Bethesda; 2016.

4. Neisser U. Cognition and reality: Principles and implications of cognitive psychology. Appleton-Croft: NY; 1967.

5. Adityanjee A, Aderibigbe YA, Theodoridis D, Vieweg VR. Dementia praecox to schizophrenia: the first 100 years. Psychiatr Clin Neurosci 1999;53:437-48.

6. Ashok AH, Baugh J, Yeragani VK. Paul Eugen Bleuler and the origin of the term schizophrenia (SCHIZOPRENIEGRUPPE). Indian J Psychiatry 2012;54(1):95-6.

7. Tsoi DT, Porwal M, Webster AC. Interventions for smoking cessation and reduction in individuals with schizophrenia. Cochrane Database of Systematic Reviews 2013;2.

8. Goff DC, Henderson DC, Amico E. Cigarette smoking in schizophrenia: relationship to psychopathology and medication side effects. Am J Psychiatry 1992;149(9):1189-94.

9. Keltner NL, Grant JS. Smoke, smoke, smoke that cigarette. Perspect Psychiatr Care 2006;42(4):256-61.

10. Breese CR, Lee MJ, Adams CE, Sullivan B, Logel J, Gillen KM, Marks MJ, Collins AC, Leonard S. Abnormal regulation of high affinity nicotinic receptors in subjects with schizophrenia. Neuropsychopharmacology 2000;23(4):351-64.

11. McCloughen A. The association between schizophrenia and cigarette smoking: a review of the literature and implications for mental health nursing practice. Int J Ment Health Nurs 2003;12(2):119-29.

12. Horst WD, Klein MW, Williams D, Werder SF. Extended use of nicotine replacement therapy to maintain smoking cessation in persons with schizophrenia. Neuropsychiatr Dis Treat 2005;1(4):349-55.

13. Kumari V, Postma P. Nicotine use in schizophrenia: the self medication hypotheses. Neurosci Biobehav Rev 2005;29(6):1021-34.

14. Kendler KS, Lönn SL, Sundquist J, Sundquist K. Smoking and schizophrenia in population cohorts of Swedish women and men: a prospective co-relative control study. Am J Psychiatry 2015;172(11):1092-100.

15. Ziedonis DM, Kosten TR, Glazer WM, Frances RJ. Nicotine dependence and schizophrenia. Psychiatr Serv 1994;45(3):204-6.

16. Ziedonis DM, George TP. Schizophrenia and nicotine use: report of a pilot smoking cessation program and review of neurobiological and clinical issues. Schizophr Bull 1997;23(2):247-54.

17. Kay SR, Fiszbein A, Opler LA. The positive and negative syndrome scale (PANSS) for schizophrenia. Schizophr Bull 1987;13(2):261-76.

18. Nasreddine ZS, Phillips NA, Bédirian V, Charbonneau S, Whitehead V, Collin I, Cummings JL, Chertkow $\mathrm{H}$. The Montreal Cognitive Assessment, MoCA: a brief screening tool for mild cognitive impairment. J Am Geriatric Soc 2005;53(4):695-9.

19. Saxena S, Chandiramani K, Bhargava R. WHOQOL-Hindi: A questionnaire for assessing quality of life in health care settings in India. Natl Med J India 1998;11(4):160-5.

20. Beck AK, Baker AL, Todd J. Smoking in schizophrenia: cognitive impact of nicotine and relationship to smoking motivators. Schizophr Res: Cognition 2015;2(1):26-32.

21. D'Souza DC, Esterlis I, Carbuto M, Krasenics M, Seibyl J, Bois F, Pittman B, Ranganathan M, Cosgrove K, Staley J. Lower $\beta 2 *$-nicotinic acetylcholine receptor availability in smokers with schizophrenia. Am J Psychiatry 2012;169(3):326-34.

22. Vanable PA, Carey MP, Carey KB, Maisto SA. Smoking among psychiatric outpatients: relationship to substance use, diagnosis, and illness severity. Psychol Addict Behav 2003;17(4):259-65.

23. Lyon ER. A review of the effects of nicotine on schizophrenia and antipsychotic medications. Psychiatr Serv 1999;50(10):1346-50.

24. Freedman R. $\alpha 7$-nicotinic acetylcholine receptor agonists for cognitive enhancement in schizophrenia. Ann Rev Med 2014;65:245-61.

25. Gilbert DG. Smoking: Individual differences, psychopathology, and emotion. Taylor \& Francis; 2014.

26. Laursen TM, Nordentoft M, Mortensen PB. Excess early mortality in schizophrenia. Ann Rev Clin Psychol 2014; 10:425-48.

27. Fujino J, Hirose K, Tei S, Kawada R, Tsurumi K, Matsukawa N, Miyata J, Sugihara G, Yoshihara Y, Ideno T, Aso T. Ambiguity aversion in schizophrenia: An fMRI study of decision-making under risk and ambiguity. Schizophr Res 2016;178(1-3):94-101.

28. de Leon J, Diaz FJ. A meta-analysis of worldwide studies demonstrates an association between schizophrenia and tobacco smoking behaviors. Schizophr Res 2005;76(2-3):135-57.

Acknowledgements - Nil ; Conflict of Interest - Nil; Funding - Nil 